\title{
Aging Differentially Affects the Re-entrainment Response of Central and Peripheral Circadian Oscillators
}

\author{
Michael T. Sellix, ${ }^{1 *}$ Jennifer A. Evans, ${ }^{2 \star}$ Tanya L. Leise, ${ }^{3}$ Oscar Castanon-Cervantes, ${ }^{2}$ DiJon D. Hill, ${ }^{2}$ Patrick DeLisser, ${ }^{2}$ \\ Gene D. Block, ${ }^{4}$ Michael Menaker, ${ }^{5}$ and Alec J. Davidson ${ }^{2}$ \\ ${ }^{1}$ Department of Medicine, Division of Endocrinology and Metabolism, University of Rochester School of Medicine and Dentistry, Rochester, New York \\ 14642, 2Department of Neurobiology, Morehouse School of Medicine, Atlanta, Georgia 30310, ${ }^{3}$ Department of Mathematics, Amherst College, Amherst, \\ Massachusetts 01002, ${ }^{4}$ Department of Psychiatry and Biobehavioral Sciences, University of California, Los Angeles, Los Angeles, California 90024, and \\ ${ }^{5}$ Department of Biology, University of Virginia, Charlottesville, Virginia 22904
}

Aging produces a decline in the amplitude and precision of $24 \mathrm{~h}$ behavioral, endocrine, and metabolic rhythms, which are regulated in mammals by a central circadian pacemaker within the suprachiasmatic nucleus (SCN) and local oscillators in peripheral tissues. Disruption of the circadian system, as experienced during transmeridian travel, can lead to adverse health consequences, particularly in the elderly. To test the hypothesis that age-related changes in the response to simulated jet lag will reflect altered circadian function, we examined re-entrainment of central and peripheral oscillators from young and old PER2::luciferase mice. As in previous studies, locomotor activity rhythms in older mice required more days to re-entrain following a shift than younger mice. At the tissue level, effects of age on baseline entrainment were evident, with older mice displaying earlier phases for the majority of peripheral oscillators studied and later phases for cells within most SCN subregions. Following a $6 \mathrm{~h}$ advance of the light:dark cycle, old mice displayed slower rates of re-entrainment for peripheral tissues but a larger, more rapid SCN response compared to younger mice. Thus, aging alters the circadian timing system in a manner that differentially affects the re-entrainment responses of central and peripheral circadian clocks. This pattern of results suggests that a major consequence of aging is a decrease in pacemaker amplitude, which would slow re-entrainment of peripheral oscillators and reduce SCN resistance to external perturbation.

\section{Introduction}

Circadian programming of behavior and physiology in mammals is subserved by a hierarchical network of biological clocks (Mohawk et al., 2012). The central circadian pacemaker, the suprachiasmatic nucleus (SCN), synchronizes (entrains) to the solar day via a retinal projection and entrains peripheral tissues through both neural and humoral cues. The molecular substrate for circadian timing is a system of interlocked transcriptional-translational feedback loops involving a family of interacting clock genes (Takahashi et al., 2008). Within the core loop, the transactivators bmall and clock drive the transcription of the repressors period (per1, per2) and cryptochrome (cry1, cry2), which feedback after translation to suppress the activity of BMAL1:CLOCK. The precise regulation of internal coordination at the molecular and systems levels is

\footnotetext{
Received July 25, 2012; revised Sept. 13, 2012; accepted Sept. 15, 2012

Author contributions: M.T.S., O.C.-C., G.D.B., M.M., and A.J.D. designed research; M.T.S., O.C.-C., D.D.H.,P.D., and A.J.D. performed research; T.L.L. and A.J.D. contributed unpublished reagents/analytic tools; M.T.S., J.A.E., T.L.L., 0.C.-C., D.D.H., P.D., and A.J.D. analyzed data; M.T.S., J.A.E., T.L.L., O.C.-C., G.D.B., M.M., and A.J.D. wrote the paper.

This work was supported by NIH/NINDS Grant U54NS060659 (A.J.D.), NIH/NIMH GrantR01 MH56647 (M.M.), NIH/NIMH Grant R01 MH062517 (G.D.B.), NIH/NCMHD Grant S21MD000101, NIH/NCRR Grant G12-RR03034, the Georgia Research Alliance, and the NSF Center for Behavioral Neuroscience. We thank Denise Holmes, Naomi lhara, Hanting Feng, Albert Tao, Gwendolyn Yao, Hing-Kiu Chan, Stanford Photonics, and the Morehouse School of Medicine Center for Laboratory Animal Research for outstanding technical support.

${ }^{*}$ M.T.S. and J.A.E. contributed equally to this work.

Correspondence should be addressed to Dr. Alec J. Davidson at Morehouse School of Medicine, 720 Westview Drive, Atlanta, GA 30310. E-mail: adavidson@msm.edu.

DOI:10.1523/JNEUROSCI.3559-12.2012

Copyright $\odot 2012$ the authors $\quad 0270-6474 / 12 / 3216193-10 \$ 15.00 / 0$
}

important for circadian and physiological function (Takahashi et al., 2008).

Transmeridian travel and shift work produce misalignment between the circadian system and the external environment ( $\mathrm{Ar}$ endt, 2009; Morris et al., 2012). Simulated jet lag also disrupts circadian organization due to differential rates of re-entrainment among different oscillatory tissues (Yamazaki et al., 2000, 2002; Davidson et al., 2008) and among different SCN subregions (Nagano et al., 2003; Nakamura et al., 2005; Davidson et al., 2009; Rohling et al., 2011). Altered temporal organization likely contributes to the severe health consequences that manifest under conditions of circadian disruption, including increased cancer risk, metabolic disorder, cognitive deficits, reduced fertility, and deregulated immune responses (Conlon et al., 2007; Scheer et al., 2009; Castanon-Cervantes et al., 2010; Mahoney, 2010; Karatsoreos et al., 2011). Notably, chronic circadian disruption also markedly increases mortality in old mice (Davidson et al., 2006).

The adverse effects of jet lag on older populations may reflect widespread age-related changes in circadian function. In both humans and animal models, senescence of the circadian system is characterized by imprecise and fragmented overt rhythms, decreased photic resetting, longer latency to re-entrain under simulated jet lag protocols, and altered function of SCN and peripheral oscillators (Li and Satinoff, 1995; Turek et al., 1995; Valentinuzzi et al., 1997; Duncan et al., 2001; Yamazaki et al., 2002; Monk, 2005; Nakamura et al., 2011; Farajnia et al., 2012). Despite evidence for adverse responses to circadian disruption in 
old animals (Davidson et al., 2006), little is known about how aging affects re-entrainment of circadian oscillators. Using bioluminescence reporters of perl expression, we have shown that old rats display tissue-specific changes in the response to simulated jet lag (Davidson et al., 2008). While noteworthy, these data were limited to a relatively small window of time following the shift, and the use of tissue-level recordings precluded investigation into how aging affects SCN spatiotemporal organization during re-entrainment.

To investigate the potential basis of age-dependent health consequences of simulated jet lag, here we test the hypothesis that aging alters re-entrainment of circadian oscillators. We exposed young (3-6 month) and old (22-28 month) PER2::luciferase (PER2::LUC) knock-in mice to a simulated jet lag protocol (6 h advance of the light:dark (LD) cycle) to assess re-entrainment responses of the SCN and four peripheral oscillators. Tissuedependent effects of age on the PER2::LUC peak time of SCN and peripheral tissues were observed during baseline and reentrainment. A detailed analysis of $\mathrm{SCN}$ re-entrainment using novel mapping methods (Evans et al., 2011) reveals that SCN organization during re-entrainment is adversely affected by aging. Overall, our data demonstrate that aging is associated with altered re-entrainment of the SCN and peripheral tissues, which likely reflect both reduced amplitude and functional output of the circadian pacemaker.

\section{Materials and Methods \\ Mice}

Homozygous PER2::LUC knock-in mice (Yoo et al., 2004), generated on a C57BL/6 background, were bred and raised under a $24 \mathrm{~h} \mathrm{LD} \mathrm{cycle}$ with $12 \mathrm{~h}$ of light and $12 \mathrm{~h}$ of darkness (LD12:12, lights-on: $0600 \mathrm{EST}$ ). After weaning, animals were group-housed in cages without running wheels. Throughout life, ambient temperature was maintained at $22 \pm 2^{\circ} \mathrm{C}$, and animals had ad libitum access to water and food (Purina Rodent Chow \#5001). All procedures were conducted according to the NIH Guide for the Care and Use of Animals and were approved by the Institutional Animals Care and Use Committee at both the University of Virginia (Charlottesville, VA) and the Morehouse School of Medicine (Atlanta, GA).

\section{Simulated jet lag protocol}

Male PER2::LUC mice (Young: 3-6 months; Old: 22-28 months) were transferred to individual cages housed within light- and temperaturecontrolled secondary enclosures and maintained under the colony LD cycle for at least 14 days. A single unidirectional shift was accomplished by advancing the time of lights-on by $6 \mathrm{~h}$ (Fig. $1 A$ ). Mice were sacrificed for tissue collection on the day before the shift (Day0, baseline day) and Day1, Day3, Day5, Day8, or Day14 after the shift. A subset of young and old mice not used for tissue collection was housed in wheel-running cages and exposed to the $6 \mathrm{~h}$ phase advance to assess age-related differences in the rate of behavioral re-entrainment (Fig. $1 \mathrm{~A}$ ). Additional mice from each age group were exposed to 8 consecutive weekly $6 \mathrm{~h}$ phase advances of the LD cycle before being sacrificed on Day0, Day1, or Day5 of the final (eighth) advance.

\section{Tissue culture}

Mice were sacrificed with $\mathrm{CO}_{2}$ asphyxiation/cervical dislocation 1-4 h before lights-off (Zeitgeber time (ZT) 8-11, where ZT12 = lights-off), as in (Davidson et al., 2009). Brains were removed, bathed in chilled Hank's solution, and sectioned in the coronal plane with a motorized vibratome at a thickness of $150 \mu \mathrm{m}$. One slice was selected from the rostrocaudal center of the SCN and trimmed near the edges of the nucleus by hand with a scalpel. Peripheral tissues (lung, thymus, spleen, and esophagus) were excised, placed in chilled Hank's solution, and trimmed by hand with a scalpel. These specific peripheral tissues were selected because they represent a cross-section of multiple physiological systems, and their phase is not reset by culture preparation (Davidson et al., 2009). All tissues were cultured on a membrane with $1.2 \mathrm{ml}$ of air-buffered medium containing $0.1 \mathrm{~mm}$ beetle luciferin. Bioluminescence rhythms from peripheral tissues were measured with a luminometer (Lumicycle; Actimetrics) housed inside a light-tight $36.5^{\circ} \mathrm{C}$ environmental chamber. SCN slices were imaged using a Stanford Photonics MEGA-10Z cooled intensified CCD camera at 15 frames/s, filtered in real time to eliminate singleimage noise events (i.e., cosmic rays), and stored as consecutive $60 \mathrm{~s}$ summed images. A $2 \mathrm{~h}$ moving average was applied (Piper Software), images were converted to $8 \mathrm{bit}$, summed to produce a series of $30 \mathrm{~min}$ images, and pixel dimensions were halved (ImageJ Software).

\section{Data analysis}

\section{Behavioral analyses}

Wheel running rhythms were monitored and analyzed with the ClockLab data collection and analysis system (Actimetrics). Starting the day after transfer to wheel-running cages, the clock times of activity onset and offset were determined, from which daily activity duration and midpoint were calculated. Activity onset was identified as the first bin above a threshold of 5 counts preceded by at least $2 \mathrm{~h}$ of inactivity and followed by at least $1 \mathrm{~h}$ of activity likewise above threshold. Activity offset was determined by a similar but opposite rule. Phase angle of entrainment was calculated as the difference between the time of lights-off and the time of activity onset. After the $6 \mathrm{~h}$ advance of the LD cycle, the number of days to re-entrain was defined for each animal as the number of days required to shift activity midpoint by $6 \pm 0.25 \mathrm{~h}$.

\section{Luminometry analyses}

Bioluminescence recordings were corrected for ZT start time, detrended by subtracting the $24 \mathrm{~h}$ running average from the raw data, and then smoothed with a $2 \mathrm{~h}$ running average (Fig. $2 \mathrm{~A}$ ) as in Davidson et al. (2008). Peak time of PER2::LUC expression was defined as the first peak in the smoothed data after $24 \mathrm{~h}$ in vitro. To evaluate re-entrainment kinetics, peak time of each tissue was plotted relative to the baseline peak time displayed on Day0 (set to 0).

\section{Bioluminescence imaging analyses}

PER2::LUC expression was mapped and analyzed using a variety of Matlab-based scripts, as described by Evans et al. (2011).

Whole SCN analyses. A time series was generated for a hand-drawn region encompassing the entire SCN (referred to as the "field rhythm"; ImageJ Software), corrected for ZT start time, and analyzed as described above (see Luminometry analyses).

Individual SCN phase maps. A time series was generated for each 12 pixel diameter region spanning the entire image (2-pixel spacing) and each time series was retained if the autocorrelation coefficient with $24 \mathrm{~h}$ lag was significant at $\alpha=0.05$, a local maximum occurred between 18 and $30 \mathrm{~h}$, and the signal-to-noise ratio was $\geq 1$. Peak time was determined from the detrended time series on the first cycle in vitro and expressed relative to projected ZT12 in vitro.

Average SCN phase maps. A representative slice was selected to which all other slices were aligned for processing. Given the high midline symmetry in PER2::LUC expression patterns (Evans et al., 2011), the right lobe was mirrored and both lobes from each sample were superimposed.

SCN cellular analyses. To locate and extract data from cell-like regions of interest (ROIs), an iterative process was employed after background and local noise subtraction (Evans et al., 2011). Based on SCN phase mapping results (see Fig. 2), cell-like ROIs within four discrete spatial subregions corresponding to the dorsal, lateral, medial, and ventral portions of the SCN (abbreviated as $\mathrm{dSCN}, 1 \mathrm{SCN}, \mathrm{mSCN}$, and $\mathrm{vSCN}$, respectively) were selected for cellular analyses of PER2::LUC peak time, period, amplitude, and brightness (Evans et al., 2011).

\section{Statistical analyses}

Statistical analyses were performed with JMP software (SAS Institute). Student's $t$ test was used to assess age-related differences in behavioral entrainment and rate of re-entrainment. To assess differences in baseline entrainment of PER2::LUC rhythms, we used full factorial ANOVA (main factors: age and tissue type or SCN subregion), followed by full 

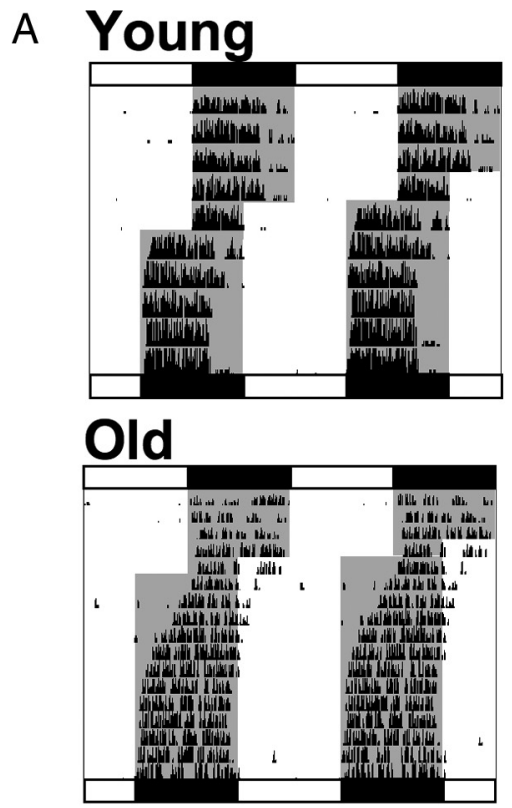
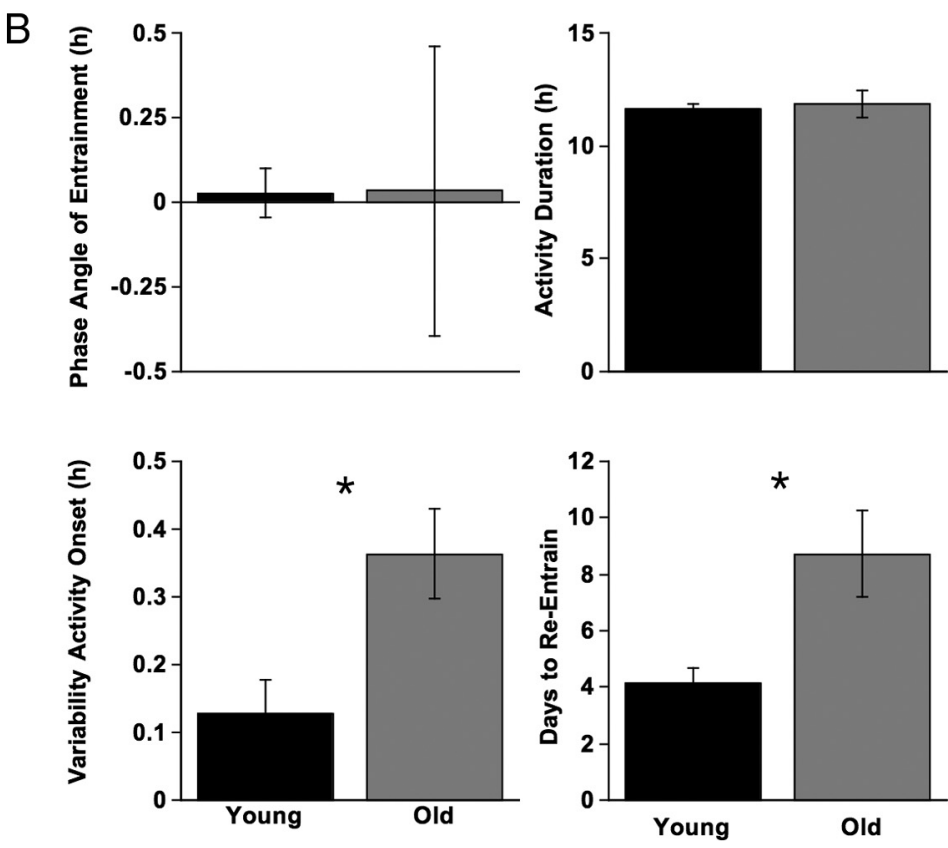

Figure 1. Effects of aging on locomotor activity rhythms under baseline conditions and during simulated jet lag. $\boldsymbol{A}$, Representative double-plotted actograms illustrating wheel-running activity of a young and old mouse that re-entrained in 4 and 8 days, respectively. White and black bars above and below each actogram depict lighting conditions in place before and after the $6 \mathrm{~h}$ advance, respectively. Gray shading in each actogram indicates the presence of darkness. $\boldsymbol{B}$, Measures of baseline activity rhythms and rate of re-entrainment in young $(n=9)$ and old $(n=7)$ mice. 0 ld mice displayed more variability in the time of activity onset and a longer latency to re-entrain following the $6 \mathrm{~h} \mathrm{LD}$ cycle advance, but they did not differ in phase angle of entrainment or activity duration. Student's $t$ test, ${ }^{*} p<0.05$.

factorial ANOVA subdivided by tissue/subregion and post hoc comparisons using least square means (LSM) contrasts or Tukey's honestly significant difference (HSD). To assess differences in re-entrainment of PER2::LUC rhythms, we used full factorial ANOVA (main factors: age, day, and tissue type, or SCN subregion), followed by full factorial ANOVA subdivided by tissue/subregion (main factors: age, day) and post hoc comparisons using LSM contrasts. For cellular analyses, a more conservative $\alpha$ level was adopted ( $\alpha<0.005)$ to avoid spurious results due to the large number of cells extracted (average number across regions and groups: 107 cell-like ROIs, with a SD of 33 cell-like ROIs). Data are represented in figures and text as mean \pm SEM.

\section{Results}

Locomotor activity rhythms

Young and old mice did not differ in phase angle of entrainment (Fig. $1 ; t$ test, $t_{(14)}=0.02, p=0.99$ ) or activity duration (Fig. 1; $\left.t_{(14)}=0.35, p=0.73\right)$, although older mice displayed more variability in the time of activity onset (Fig. $1 ; t_{(14)}=2.85, p=0.013$ ). Consistent with previous results (Valentinuzzi et al., 1997), old mice showed significantly slower re-entrainment following a $6 \mathrm{~h}$ advance than their younger counterparts (Fig. $1 ; t_{(14)}=3.12, p=$ $0.008)$.

\section{Baseline entrainment}

Peripheral tissues and SCN field rhythms

Peak times of PER2::LUC during baseline varied as a function of age $\left(F_{(1,81)}=13.4, p<0.0005\right)$ and tissue type $\left(F_{(4,81)}=4.4, p<\right.$ $0.005)$, but there was not a significant interaction between these variables $\left(F_{(4,81)}=1.96, p=0.11\right)$. Relative to young mice, old mice displayed a significantly earlier peak time for lung and thymus (Fig. 2A, B; LSM contrasts, $p<0.01$ ), with a trend for an earlier peak time for the esophagus (Fig. $2 A, B$; LSM contrasts, $p<0.03)$. The baseline phase of entrainment for the SCN field rhythm and spleen did not differ by age (Fig. $2 A, B$; LSM contrasts, $p>0.5$ ).
Cell-like ROIs within SCN subregions

Peak times of PER2::LUC displayed by SCN cell-like ROIs during baseline were affected by age $\left(F_{(1,810)}=47.5, p<0.0001\right)$ and SCN subregion $\left(F_{(3,810)}=146.3, p<0.0001\right)$, with a trend for an interaction between these two factors $\left(F_{(3,810)}=4.2, p=0.006\right)$. For both young and old mice, PER2::LUC expression patterns within the SCN were similar to previous results (Evans et al., 2011), with cell-like ROIs within dSCN and vSCN subregions phase leading those within ISCN and mSCN subregions (Fig. $2 C-E)$. Relative to young mice, old mice displayed later peak times of cell-like ROIs within the vSCN, ISCN, and mSCN subregions (Fig. $2 D, E$; LSM contrasts, $p<0.005$ ), but not the dSCN subregion (Fig. $2 D, E$; LSM contrasts, $p>0.3$ ). Cellular parameters of period, amplitude, and brightness also varied between SCN subregions and age groups (Fig. 2D; Table 1). For each age group, PER2::LUC rhythm amplitude and peak expression were both greater for cell-like ROIs within the mSCN subregion (Table 1 ; Tukey's HSD, $p<0.005$ ), and cell-like ROIs within the vSCN subregion displayed shorter period lengths (Table 1; Tukey's HSD, $p<0.005)$ compared to the other SCN subregions. For each of the four SCN subregions examined, younger mice displayed lower amplitude PER2::LUC rhythms with longer period and lower peak PER2::LUC levels relative to their older counterparts (Table 1; LSM contrasts, $p<0.005$ ).

\section{Re-entrainment of PER2::LUC Rhythms}

Peripheral tissues and SCN field rhythms

Aging significantly altered re-entrainment to a $6 \mathrm{~h}$ advance of the LD cycle in a tissue-specific manner (Fig. 3). Re-entrainment response was significantly influenced by shift day $\left(F_{(4,378)}=58.3\right.$, $p<0.0001)$, age $\left(F_{(1,378)}=58.5, p<0.0001\right)$, and tissue type $\left(F_{(4,378)}=16.1, p<0.0001\right)$, with a significant interaction between these variables $\left(F_{(16,378)}=1.7, p<0.05\right)$. Among the tissues examined, only the re-entrainment response of the spleen 
A

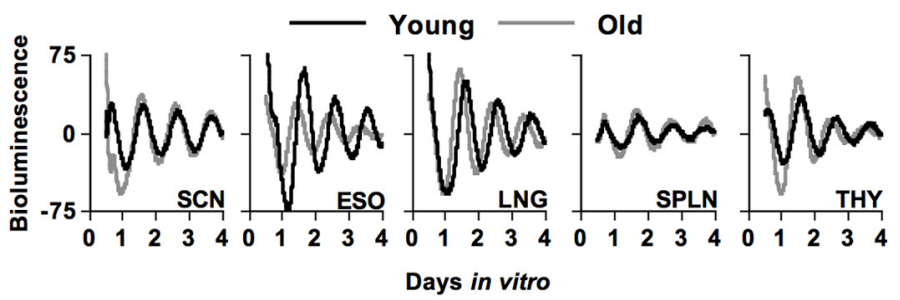

B

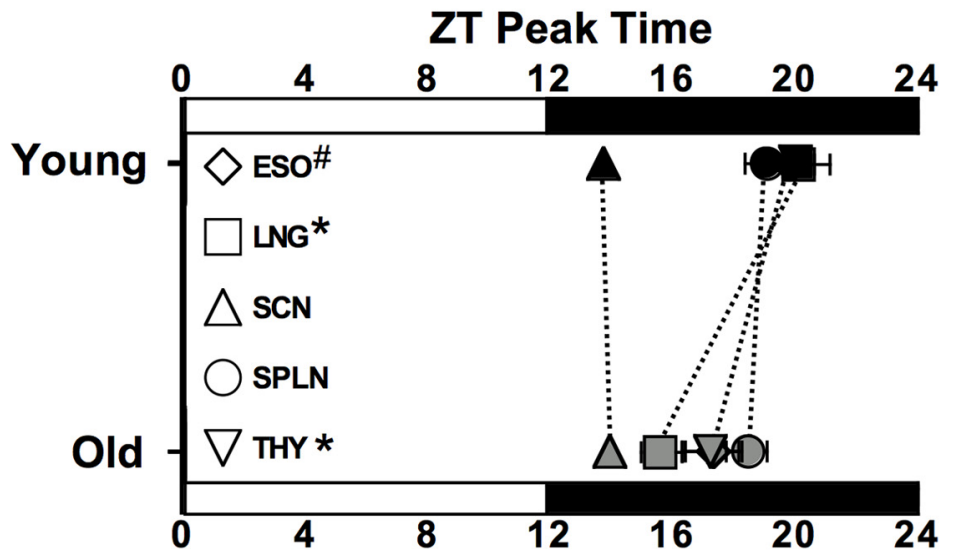

C

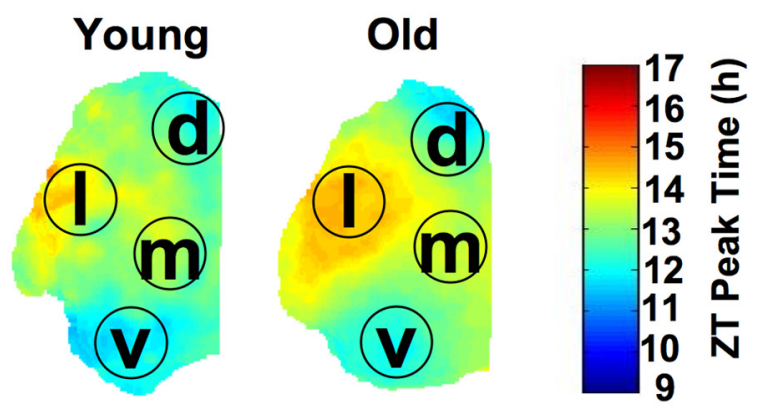

D

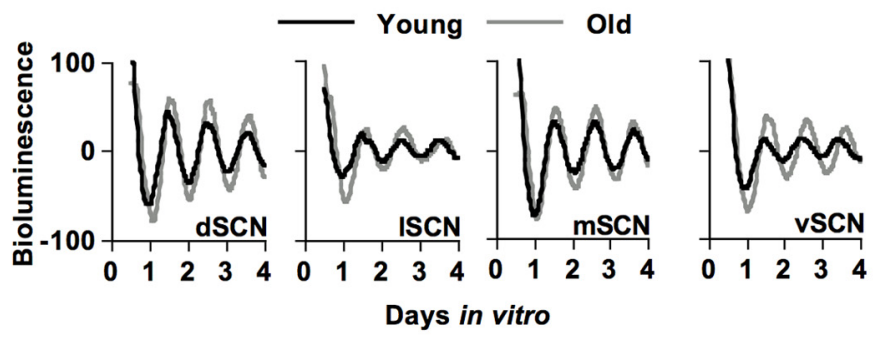

$E$

ZT Peak Time

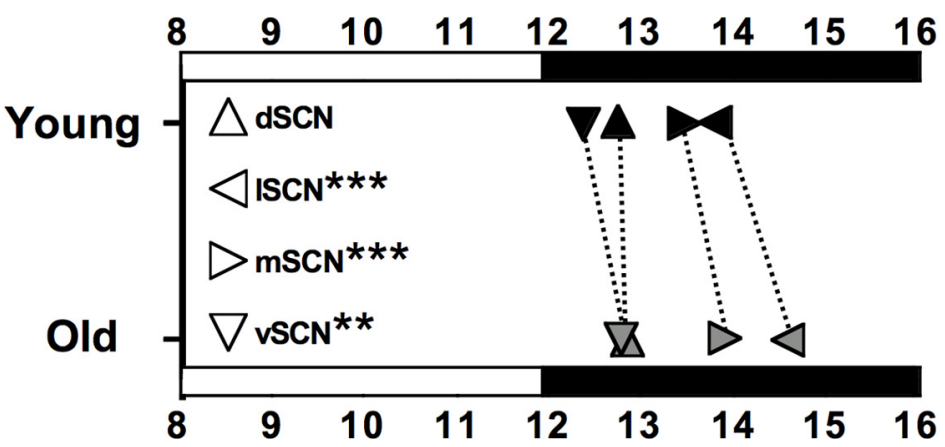

Figure 2. Effects of age on PER2::LUC rhythms of central and peripheral oscillators under baseline conditions (Day0). A, Representative time series of PER2::LUC rhythms displayed by SCN and peripheral oscillators from young and old mice. was not affected by age (Fig. $3 ; F_{(1,92)}=$ $1.3, p=0.25)$. In older mice, the esophagus and thymus took substantially longer to re-entrain relative to tissues extracted from young mice (Fig. 3; esophagus: $F_{(1,80)}=45.2, p<0.0001$; thymus: $F_{(1,88)}$ $=17.3, p<0.0001)$. Likewise, the lung of older mice displayed smaller phase advances relative to the lung of young mice, with the latter re-entraining quickly but with altered phase (Fig. 3; $F_{(1,96)}=90.1$, $p<0.0001$ ).

Re-entrainment of the SCN field rhythm was also affected by age, but in a manner distinct from that of peripheral tissues (Fig. 3; $F_{(1,22)}=5.3, p<0.05$ ). SCN of older mice displayed a large phase advance on Day3 after the $6 \mathrm{~h}$ advance of the LD cycle and overshot the target phase on Day5 before stably aligning into a more typical phase of re-entrainment by Day8 (Fig. 3). In contrast, the SCN of younger animals displayed a more linear pattern of re-entrainment, but also appeared to display a slight overshoot of the target phase on Day14 (Fig. 3).

Cell-like ROIs within SCN subregions

Dynamic changes in SCN spatiotemporal organization were observed in slices from both young and old mice (Fig. 4), with agerelated differences evident. Re-entrainment of cell-like ROIs within $\mathrm{SCN}$ subregions was affected by shift day $\left(F_{(4,2909)}=623.1, p<\right.$ $0.0001)$, age $\left(F_{(1,2909)}=217.6, p<0.0001\right)$, and SCN subregion $\left(F_{(3,2909)}=83.2, p<\right.$ $0.0001)$, with a significant interaction between these variables $\left(F_{(12,2909)}=8.5, p<\right.$ 0.0001). SCN spatiotemporal organization

Time series are detrended and corrected for ZT start time, with the start of each recording corresponding to ZT12 on the day of culture. $\boldsymbol{B}$, Peak time of PER2::LUC rhythms for esophagus (ESO), lung (LNG), spleen (SPLN), thymus (THY), and the SCN field rhythm in young and old mice during baseline. Dashed lines connect comparable structures in young and old mice to highlight the effects of age. Relative to young mice, old mice displayed a significantly earlier peak time for lung and thymus and a trend for an earlier peak time for the esophagus. The baseline phase of entrainment of the SCN field rhythm and spleen did not differ by age. C, Average phase maps illustrate the spatial location of each $\mathrm{SCN}$ subregion analyzed: dorsal SCN (d, dSCN), lateral SCN (I, ISCN), medial SCN (m, mSCN), and ventral SCN (v, vSCN). D, Representative time series of PER2::LUC rhythms displayed by $\mathrm{SCN}$ neurons from young and old mice, plotted as in $\boldsymbol{A}$. $\boldsymbol{E}$, Peak time of PER2::LUC rhythms displayed by cell-like ROls within SCN subregions from young and old mice during baseline, plotted as in $\boldsymbol{B}$. Number of cultures per group: ESO-Young $=12$, ESO-Old $=8$; LNGYoung $=13$, LNG-Old $=8$; SCN-Young $=4$, SCN-Old $=4$; SPLN-Young $=12$, SPLN-Old $=8$; THY-Young $=14$, THYOld $=8$. Results of post hoc LSM Contrasts are illustrated adjacent to each symbol within the legend: ${ }^{* * *} p<0.0005$, ${ }^{* *} p<0.001, p<0.01$, $p<<0.05$. 
Table 1. Rhythmic parameters of cell-like ROls for each age group under baseline (Day $\mathbf{0}$ ) conditions

\begin{tabular}{|c|c|c|c|c|c|c|c|c|}
\hline & Peak time & & Period & & Amplitude & & Brightness & \\
\hline & Young & Old & Young & Old & Young & Old & Young & Old \\
\hline$d S C N$ & $13.1 \pm 0.1^{b}$ & $13.2 \pm 0.1^{c}$ & $25.29 \pm 0.12^{a, b}$ & $23.98 \pm 0.05^{* a}$ & $33 \pm 3^{a}$ & $60 \pm 3^{* a, b}$ & $52 \pm 5^{a, b}$ & $118 \pm 5^{* b}$ \\
\hline ISCN & $14.2 \pm 0.1^{a}$ & $14.9 \pm 0.1^{* a}$ & $25.48 \pm 0.17^{a}$ & $24.04 \pm 0.08^{* a}$ & $21 \pm 2^{b}$ & $57 \pm 3^{* a, b}$ & $44 \pm 4^{b}$ & $121 \pm 4^{* b}$ \\
\hline $\mathrm{mSCN}$ & $13.8 \pm 0.1^{a}$ & $14.2 \pm 0.1^{* b}$ & $25.01 \pm 0.06^{b}$ & $23.93 \pm 0.07^{* a}$ & $34 \pm 2^{a}$ & $66 \pm 3^{* a}$ & $66 \pm 4^{a}$ & $143 \pm 4^{* a}$ \\
\hline vSCN & $12.7 \pm 0.1^{b}$ & $13.1 \pm 0.1^{* c}$ & $24.87 \pm 0.13^{b}$ & $23.72 \pm 0.04^{* b}$ & $19 \pm 1^{b}$ & $52 \pm 2^{* b}$ & $41 \pm 3^{b}$ & $117 \pm 4^{* b}$ \\
\hline
\end{tabular}

${ }^{*}$ Different from identical region in young group, LSM contrast, $p<0.005$.

$a, b$, cDifferent letters represent differences between regions within each age group, Tukey's HSD, $p<0.005$.

at baseline is characterized by the dSCN and vSCN subregions phase leading the ISCN and $\mathrm{mSCN}$ subregions. On the first day after the $6 \mathrm{~h}$ LD cycle advance, the young SCN showed a similar phase distribution as that under the former LD cycle, with the exception that celllike ROIs within the vSCN subregion displayed a phase advance of 3-4 h (Fig. 4A,B). On subsequent days, we observed differential re-entrainment of two spatially distinct regions that produced a large regional phase difference on Day5 (Fig. $4 A, B$ ). On Day8, SCN regional relationships and phase angle appear to normalize and be maintained thereafter (Fig. $4 A, B$ ). Similar overall organizational changes were observed for SCN slices from old mice, but we observed clear age-dependent differences in these patterns (Fig. 4A, $B$ ). In the old SCN, the large regional phase difference was observed earlier (Day3) than their younger cohorts (Day 5). On Day5, the phase relationships among SCN regions appeared to be normal for old mice; however, the phase advance exhibited by all four SCN regions exceeded $6 \mathrm{~h}$ (Fig. $4 A, B$ ). SCN slices from old mice were stably realigned by Day8, like SCN slices from young mice. For celllike ROIs within each SCN subregion, PER2::LUC peak times were altered by age, with older mice showing larger initial shifts and differences in final phase of specific subregions (Fig. $4 B$; LSM contrasts, $p<0.005)$.

SCN from old mice also displayed greater phase dispersion among cell-like ROIs on days following the shift (Fig. 4C). The standard deviation of PER2::LUC peak times among cell-like ROIs was affected by shift day $\left(F_{(5,337)}=16.7, p<0.0001\right)$, age $\left(F_{(1,337)}=6.2, p<0.05\right)$, and SCN subregion $\left(F_{(3,337)}=19.4, p<\right.$ $0.0001)$, with a significant interaction between these variables $\left(F_{(20,337)}=2.2, p<0.005\right)$. Cell-like ROIs from old mice displayed greater phase dispersion across all SCN subregions (Fig. $4 C$; LSM contrast, $p<0.01$ ) and within the ISCN, mSCN, and vSCN subregions on specific postshift days (Fig. 4C; LSM contrasts, $p<0.01)$. Phase dispersion in the dSCN was not significantly affected by age (Fig. 4C), although old mice displayed a larger increase in phase dispersion 3-5 days after the shift.

\section{Re-entrainment responses following repeated advances of the LD cycle}

Repeated 6 h LD cycle advances (i.e., chronic simulated jet lag) produced tissue-specific effects on the re-entrainment responses of peripheral tissues, with re-entrainment responses being affected by shift day $\left(F_{(1,328)}=285.6, p<0.0001\right)$, age $\left(F_{(1,328)}=\right.$ $40.7, p<0.0001)$, and tissue type $\left(F_{(3,328)}=10.2, p<0.0001\right)$, with an interaction between tissue type and shift number $\left(F_{(1,378)}=58.5, p<0.0001\right)$. When subdivided by age and tissue type, a main effect of repeated shifts was observed for the thymus of older mice $\left(F_{(1,30)}=5.2, p<0.05\right)$ and the lung of young mice $\left(F\left(_{1,41)}=58.5, p<0.05\right)\right.$, with a trend for an effect in the esophagus of older mice $\left(F_{(1,28)}=4.1, p=0.054\right)$. For the esophagus of older mice, antidromic resetting (initial phase delay following the advance of the LD cycle), which was present on Dayl after the first shift, was no longer observed on Dayl of the eighth shift
(Fig. 5; LSM contrast, $p<0.02$ ). Antidromic resetting emerged for the thymus of old mice on Dayl of the eighth LD cycle advance, which was not evident for the first LD cycle advance (Fig. 5 ; LSM contrast, $p<0.01$ ). In young mice, the large overshoot of the lung, which was present on Day5 of the first LD cycle advance, was mitigated during the eighth LD cycle advance (Fig. 5; LSM contrast, $p<0.02$ ).

\section{Aging differentially affects rate of re-entrainment for central and peripheral oscillators}

To assess age-related changes in the rate of re-entrainment, we calculated the magnitude of the shift per cycle for the first 5 days after the $6 \mathrm{~h}$ LD cycle advance (Fig. 6). For reentrainment after the first shift of the LD cycle, younger mice displayed faster re-entrainment than their older counterparts for the esophagus (Fig. $6 A ; t_{(25)}=-4.8, p<0.0001$ ), the lung (Fig. $6 A ; t_{(27)}=-11.7, p<0.0001$ ), and the thymus (Fig. $6 A$; $t_{(26)}=-5.3, p<0.0001$ ), but not the spleen (Fig. $6 A ; t_{(28)}=$ $-1.3, p=0.19)$. Similar results were obtained for rate of re-entrainment of peripheral tissues collected after the eighth shift of the LD cycle (Fig. $6 B$; esophagus: $t_{(7)}=-3.2, p<0.05$; lung: $t_{(6)}=-3.2, p<0.05$; spleen: $t_{(5)}=0.2, p=0.86$; thymus: $\left.t_{(6)}=-2.5, p<0.05\right)$. In contrast, older mice displayed faster SCN re-entrainment compared to young mice (Fig. $6 C$; field: $t_{(8)}=3.0, p<0.05$; all: $t_{(724)}=26.8, p<0.0001$; dSCN: $t_{(156)}=$ 14.0, $p<0.0001$; 1SCN: $t_{(176)}=14.5, p<0.0001 ; \mathrm{mSCN}$ : $\left.t_{(210)}=19.4, p<0.0001 ; \mathrm{vSCN}: t_{(176)}=8.9, p<0.0001\right)$

\section{Discussion}

We have shown that aging alters re-entrainment at multiple levels of circadian organization. Consistent with previous work (Valentinuzzi et al., 1997), locomotor activity rhythms displayed by old mice required a greater number of days to re-entrain than that required by their younger counterparts. Likewise, peripheral oscillators from older PER2::LUC mice displayed tissue-specific slowing of re-entrainment responses, as in rats (Davidson et al., 2008). While the sluggish responses of peripheral tissues from old mice mirrored the slow re-entrainment at the behavioral level, the old SCN displayed larger, more rapid shifts of PER2::LUC rhythms relative to the young SCN. Concomitantly, phase dispersion among SCN neurons from old mice was increased during re-entrainment, and the old SCN displayed a large "overshoot" of the target phase before stable re-entrainment was achieved. As discussed in detail below, our results suggest that a major consequence of aging is a decrease in pacemaker amplitude that slows peripheral re-entrainment and decreases SCN resistance to external perturbation. This proposed decrease in pacemaker amplitude is not reflected in PER2::LUC amplitude, but instead is likely related to age-related declines in electrophysiological rhythms and neuropeptide production (Roozendaal et al., 1987; Duncan et al., 2001; Kalló et al., 2004; Nakamura et al., 2011; Farajnia et al., 2012). 


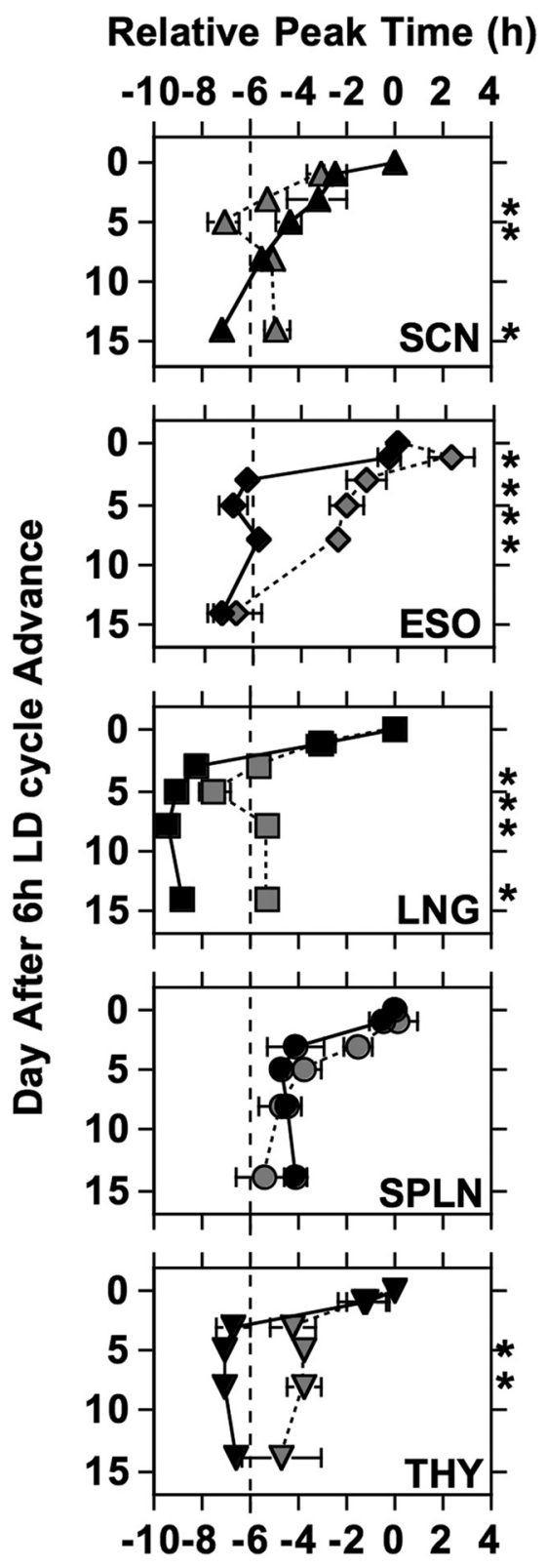

Figure 3. Re-entrainment of PER2:::LUC rhythms for the SCN field rhythm, esophagus (ESO), lung (LNG), spleen (SPLN), and thymus (THY) from young mice (black symbols) and old mice (gray symbols). For each age group, peak time of each tissue is normalized to that displayed on Day0 (set to 0). Dashed vertical line illustrates target phase after the $6 \mathrm{~h} \mathrm{LD}$ cycle advance. Number of cultures per group: ESO-Young $=12$ (Day0), 16 (Day1), 6 (Day3), 16 (Day5), 8 (Day8), 7 (Day14); ESO-Old $=8,10,6,11,6,4$; LNG-Young $=13,18,6,17,9,12$; LNG-0ld $=$ $8,10,7,12,7,8 ;$ SCN-Young $=4,2,2,4,3,3 ;$ SCN-Old $=4,4,3,4,3,4 ;$ SPLN-Young $=12,16$, $6,17,9,12 ;$ SPLN-Old $=8,10,6,3,6,7$; THY-Young $=14,16,5,16,9,10$; THY-Old $=8,10,6$, $12,7,7$. LSM contrasts, ${ }^{*} p<0.01$.

SCN spatiotemporal organization during re-entrainment To date, this is the most comprehensive imaging study of SCN re-entrainment kinetics, and our novel mapping approach reveals the precise spatial location and temporal dynamics of the differentially shifted SCN subregions indicated by previous work (Nagano et al., 2003; Nakamura et al., 2005; Davidson et al., 2009; Rohling et al., 2011). We find that the ventral SCN is the first to advance, and other regions follow suit over subsequent days. SCN spatiotemporal organization during reentrainment is reminiscent of the two functionally distinct compartments oftentimes referred to as the "shell" and "core" (Antle and Silver, 2005; Morin, 2007), despite the apparent lack of this spatiotemporal organization during baseline entrainment. Regional differences in re-entrainment likely reflect the dense retinal innervation of the ventral SCN (Antle and Silver, 2005; Han et al., 2012). While our experiments focused on a LD advance, regional phase differences also manifest in the delay direction (Nagano et al., 2003; Nakamura et al., 2005; Rohling et al., 2011). Because LD advances cause more severe health consequences than LD delays of equal magnitude, future experiments could assess whether there is direction-specific variability in the spatiotemporal pattern of SCN re-entrainment.

\section{Age-dependent changes in circadian organization} and re-entrainment

This is the first study to investigate age-related changes in SCN spatiotemporal organization. Consistent with Yamazaki et al. (2002), SCN field rhythms were similarly phased in young and old PER2::LUC mice. However, SCN region-specific effects of age were evident during baseline entrainment, with most regions in the old SCN displaying later peak times. Because several peripheral tissues are phase advanced in old mice, this suggests that aging alters circadian organization in multiple ways. SCN cells also displayed larger amplitude and increased PER2::LUC expression in older mice. Previous reports have found similar robust rhythms of per gene expression in the SCN of young and old animals (Yamazaki et al., 2002; Davidson et al., 2008; Nakamura et al., 2011), although age-related changes in other clock genes have been reported (Kolker et al., 2003; Wyse and Coogan, 2010). Aging does not appear to reduce the overall number of SCN neurons (Madeira et al., 1995; Roberts et al., 2012), but it alters specific SCN subpopulations and network properties (Roozendaal et al., 1987; Van der Zee et al., 1999; Duncan et al., 2001; Kalló et al., 2004; Palomba et al., 2008). Functional and organizational changes under steady-state entrainment conditions may have adverse consequences for physiological processes regulated by the circadian system and could contribute to functional deterioration associated with aging (Kondratova and Kondratov, 2012).

Re-entrainment of the old SCN was more erratic than the relatively linear pattern displayed by the young SCN (see also Davidson et al., 2009). In old mice, we observed an increased degree of desynchrony on the third day after the shift, with a substantial phase advance of the vSCN. This pattern was not observed in young mice until 5 days postshift. Thus, the SCN from older mice shifted more rapidly and with increased desynchrony. This contrasts with our previous study using the per1-luc rat (Davidson et al., 2008), which found an age-related slowing of SCN re-entrainment. These conflicting results may be explained by species differences, nature of the reporter strain, or differences in clock gene function (Yan and Silver, 2004).

For peripheral tissues, our results, in part, parallel those obtained with old per1-luc rats (Davidson et al., 2008). In both studies, age altered peripheral re-entrainment in a tissue-specific manner. In rats, the old liver displayed slower re-entrainment relative to the young liver, whereas the old arcuate nucleus and pineal gland were characterized by more rapid shifts. Differential effects of aging on the re-entrainment of extra-SCN neural versus peripheral tissues may reflect differences in the monosynaptic versus polysynaptic nature of SCN input (Moore, 1996; Gerhold et al., 2001; Egli et al., 2004). Furthermore, differences among peripheral tissues may reflect tissue-specific control by different temporal cues (Vujovic et al., 2008; Dibner et al., 2010) and/or 


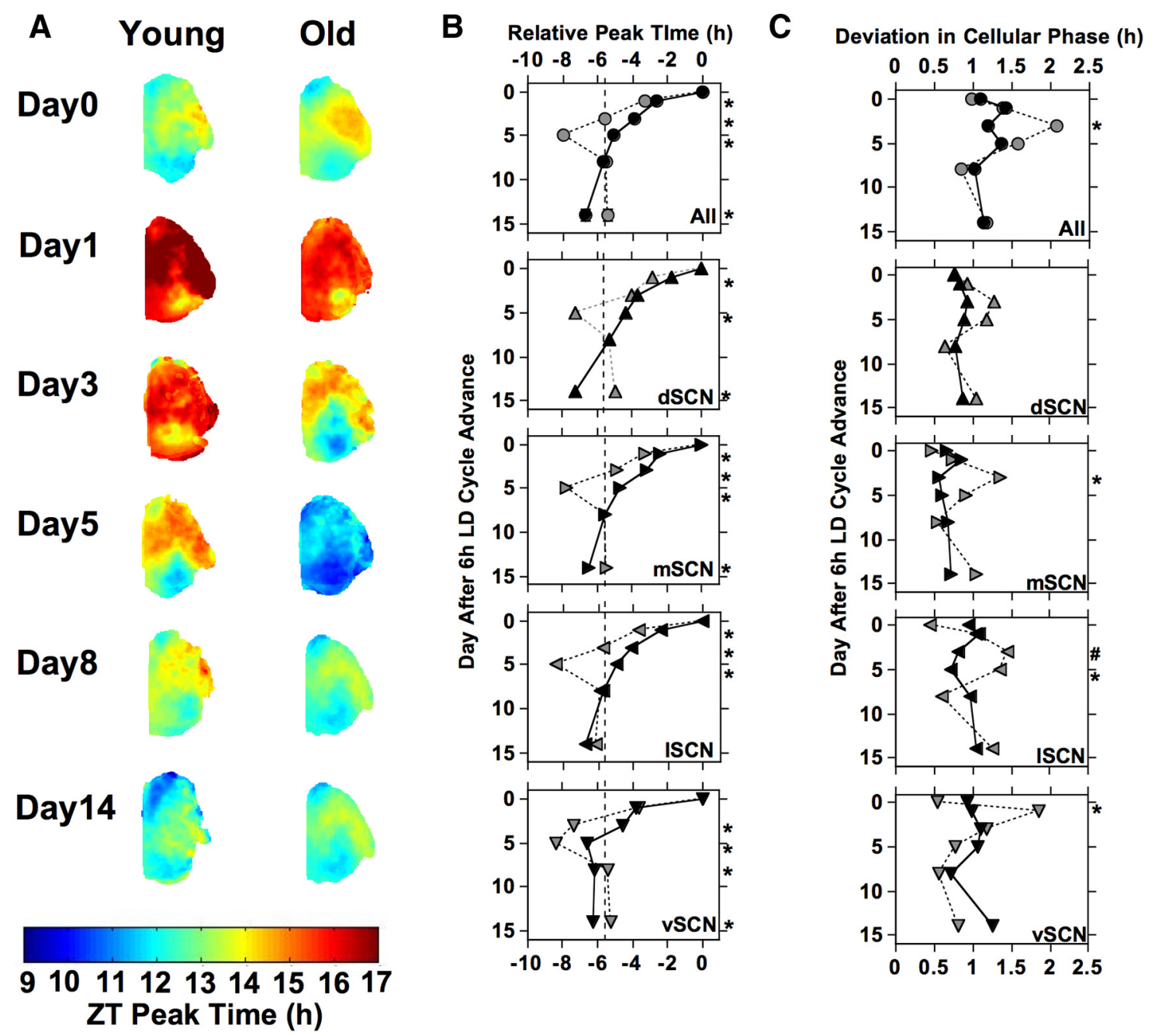

Figure 4. Re-entrainment of the SCN from young mice (black symbols) and old mice (gray symbols). A, Average phase maps illustrating age-related changes in SCN spatiotemporal organization during re-entrainment. $\boldsymbol{B}$, Re-entrainment PER2::LUC rhythms of cell-like ROIs pooled across all subregions ("All”) and within each individual subregion. For each age group, peak time is normalized to that displayed on DayO (set to 0). C, Standard deviation of PER2::LUC peak time during re-entrainment for cell-like ROls pooled across all subregions ("All") and within each individual subregion. LSM contrasts, ${ }^{*} p<0.005 .{ }^{\#} p=0.012$.

different SCN subregions (Gerhold et al., 2001; Kalsbeek et al., 2006).

Putative mechanisms of age-related differences in re-entrainment response

Most of the peripheral tissues investigated here entrained more slowly in old mice, which may be due to an age-related decline in the strength of SCN outputs. In particular, aging decreases the amplitude of SCN rhythms in electrical firing and neuropeptide production (Roozendaal et al., 1987; Kalló et al., 2004; Nakamura et al., 2011), which likely contributes to lower amplitude rhythms in downstream tissues and at the overt level (Wise et al., 1994; Li and Satinoff, 1995; Turek et al., 1995; Yamazaki et al., 2002; Nakamura et al., 2011). An age-dependent decrease in SCN outputs may be sufficient to account for slower re-entrainment in peripheral tissues, but aging could also diminish the responsiveness of peripheral oscillators to SCN cues.

Older mice displayed slower re-entrainment of behavior and peripheral oscillators, but faster SCN re-entrainment of PER2::LUC rhythms relative to younger cohorts. This may seem counterintuitive, especially given work indicating that age diminishes SCN responses to light (Kolker et al., 2003; Lupi et al., 2012). However, reduced pacemaker amplitude can likewise account for the accelerated response of the old SCN, since weak or damped oscillators respond more robustly to an external perturbation (Pulivarthy et al., 2007; Abraham et al., 2010). One caveat is that culture preparation can affect the phase of some cultured tissues in the perl-luc rat (Yoshikawa et al., 2005). The SCN of young PER2::LUC mice is not reset by culture (Davidson et al., 2009), although the age-related decline in pacemaker amplitude could sensitize the SCN. However, this explanation of the current findings is unlikely, since the days on which the SCN of old mice displayed the largest phase shifts were not those with the largest discrepancy between dissection time and peak PER2::LUC. In either case, our results suggest that aging decreases pacemaker amplitude, the mechanistic basis of which may involve changes at the electrophysiological level (Biello, 2009; Farajnia et al., 2012; Nakamura et al., 2011) and/or dissociation between molecular and membrane events (Vansteensel et al., 2003; Nakamura et al., 2011). Alternatively, the current findings may relate to network-level changes that alter synchrony among SCN neurons, since pacemaker amplitude does not al- 


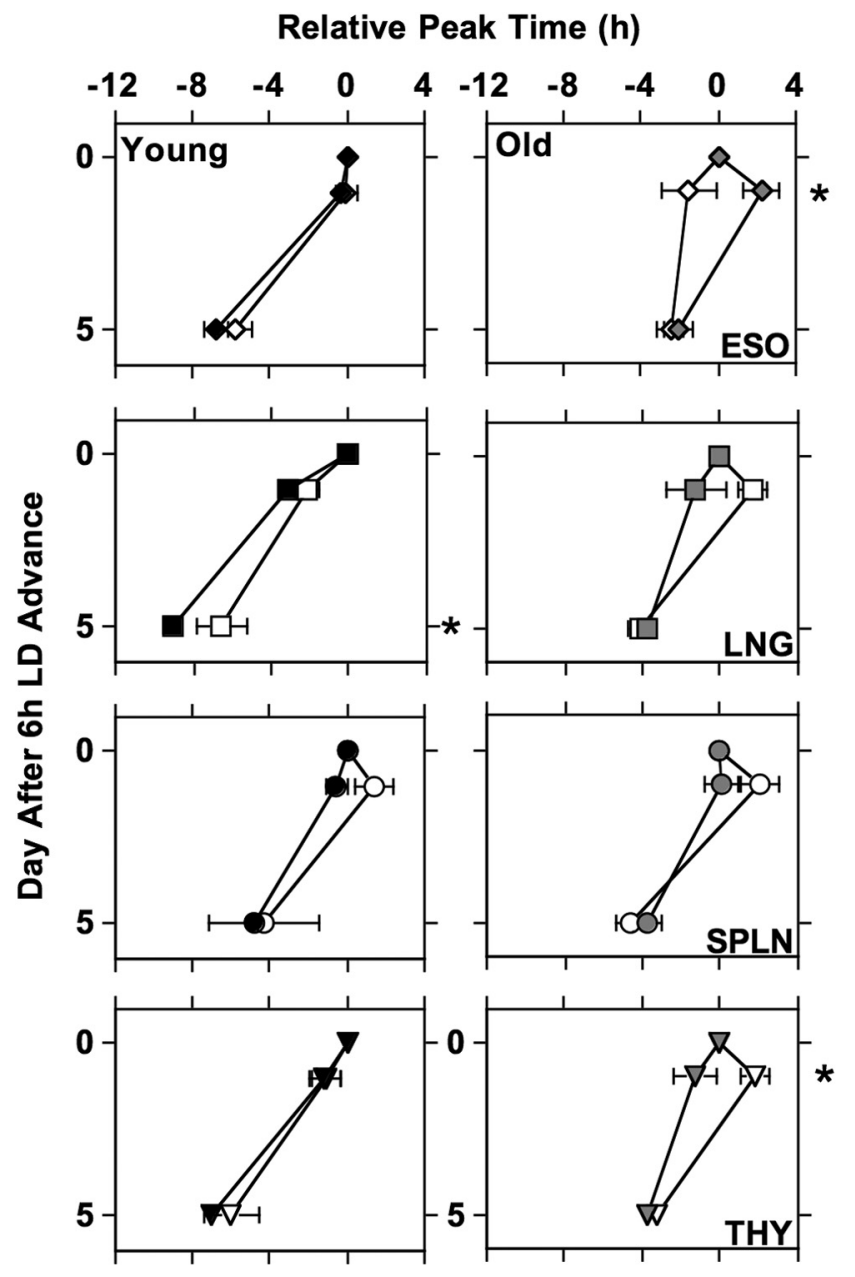

Figure 5. Re-entrainment responses of peripheral tissues during the first (filled symbols) and eighth (open symbols) $6 \mathrm{~h} \mathrm{LD}$ cycle advance. Number of cultures per group: esophagus (ES0)-Young = 12 (Shift1-Day0), 16 (Shift1-Day1), 16 (Shift1-Day5), 4 (Shift8-Day0), 7 (Shift8-Day1), 4 (Shift8-Day5); ESO-Old = 8, 10, 11, 5, 6, 5; lung (LNG)-Young = 13, 18, 17, 4, 6,4 ; LNG-Old $=8,10,12,6,7,5$; spleen (SPLN)-Young $=12,16,17,2,4,2$; SPLN-Old $=8,10$, $13,6,6,5$; thymus (THY)-Young $=14,16,16,4,6,3 ;$ THY-Old $=8,10,12,6,7,5$. LSM contrasts, ${ }^{*} p<0.01$.

ways account for phase-shifting responses, and intercellular synchronization at the electrophysiological level can influence resetting responses (vanderLeest et al., 2009).

Paradoxically, young mice displayed rapid re-entrainment of behavior and peripheral tissues, but relatively slow SCN reentrainment. While it remains unclear how peripheral tissues could respond faster than the SCN, this may relate to the fact that the SCN and peripheral oscillators can respond differently to resetting cues (Vujovic et al., 2008; Buhr et al., 2010; Sujino et al., 2012). Acute changes in light and darkness can alter the overt expression/levels of locomotor activity, body temperature, hormone release, and feeding (Mrosovsky, 1999), which may facilitate re-entrainment of peripheral oscillators that are more sensitive to these cues than the SCN. Because age decreases the amplitude of overt rhythms, this influence is likely diminished in old animals and does not facilitate re-entrainment.

We also examined the re-entrainment of peripheral tissues following eight consecutive phase advances. There did not appear to be an overall additive effect of multiple shifts in either age group despite compelling evidence that chronic circadian disruption has adverse health consequences (Davidson et al., 2006; Castanon-Cervantes et al., 2010). If anything, specific peripheral
A
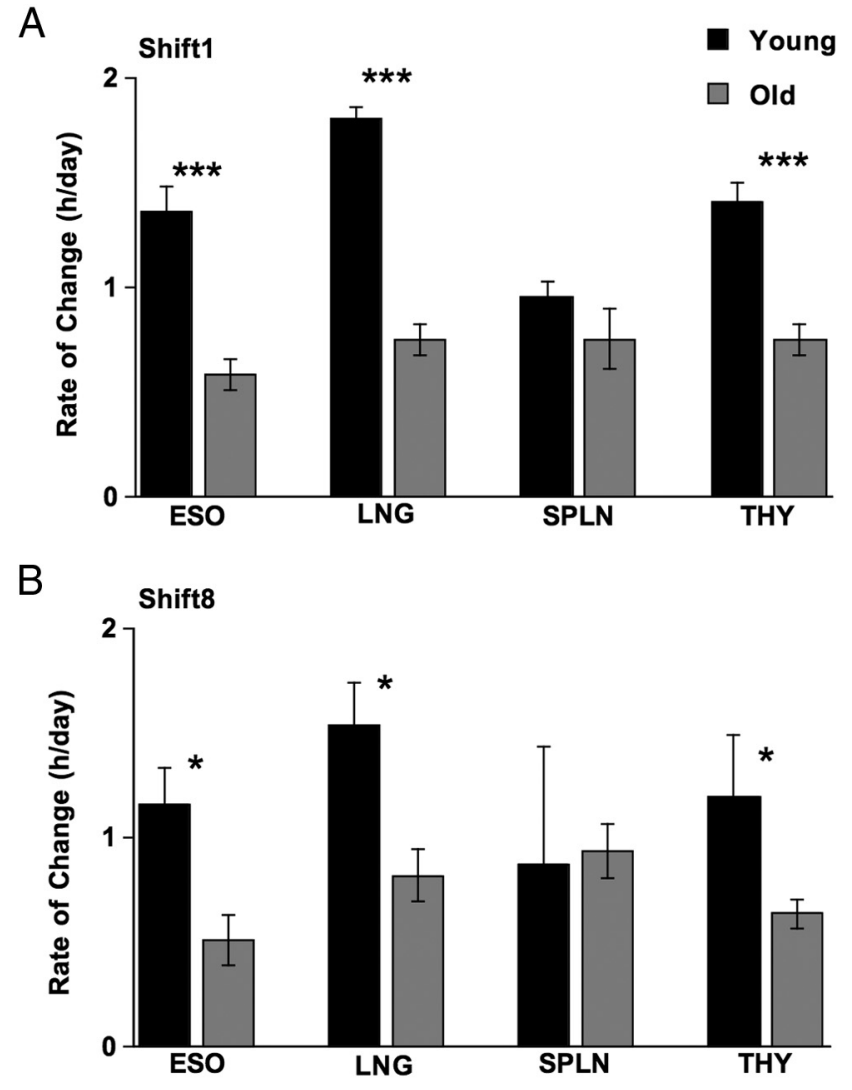

C

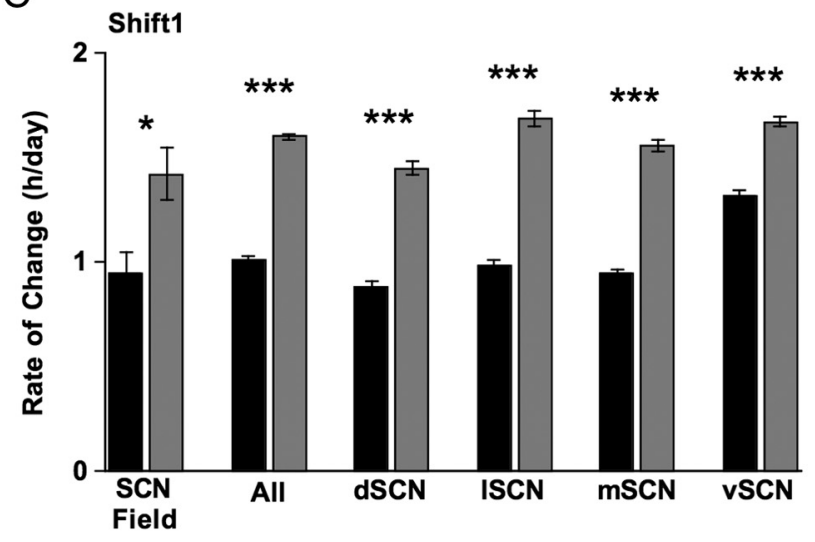

Figure 6. Aging differentially affects rate of re-entrainment for central and peripheral oscillators. To assess rate of re-entrainment, the normalized phase shift displayed on Day5 was divided by the number of days after the $6 \mathrm{~h} \mathrm{LD}$ cycle advance. $A$, Rate of re-entrainment for peripheral tissues after the first shift of the LD cycle. $\boldsymbol{B}$, Rate of re-entrainment for peripheral tissues after the eighth shift of the LD cycle. C, Rate of re-entrainment for the SCN field rhythm, cell-like ROIs pooled across all SCN subregions ("All"), and within each individual SCN subregion after the first shift of the LD cycle. Student's $t$ test, ${ }^{* * *} p<0.0001,{ }^{* *} p<0.005,{ }^{*} p<0.05$. ESO, Esophagus; LNG, lung; SPLN, spleen; THY, thymus.

tissues displayed an improvement in re-entrainment response, which may support previous work indicating that the circadian system displays adaptive plasticity during chronic jet lag (Yan, 2011). However, peripheral tissues from old mice displayed consistently slower re-entrainment, which likely produces cumulative effects with long-term health consequences.

\section{Summary and Conclusions}

Our results demonstrate that aging affects re-entrainment of central and peripheral circadian oscillators in a tissue-dependent 
manner. We propose that these age-related changes reflect a decline in pacemaker amplitude that would have consequences for SCN function and the systems under its control. Continued investigation of how age affects the circadian timing system can provide insight into the circadian disorders frequently observed in elderly populations (Mazza et al., 2004; Ancoli-Israel and Ayalon, 2006). Future experiments can also assess the effectiveness of chronobiological treatments for promoting re-entrainment, health, and longevity in the elderly (van Gool and Mirmiran, 1986; Lucassen et al., 1995; Gubin et al., 2006).

\section{References}

Abraham U, Granada AE, Westermark PO, Heine M, Kramer A, Herzel H (2010) Coupling governs entrainment range of circadian clocks. Mol Syst Biol 6:438.

Ancoli-Israel S, Ayalon L (2006) Diagnosis and treatment of sleep disorders in older adults. Am J Geriatr Psychiatry 14:95-103.

Antle MC, Silver R (2005) Orchestrating time: arrangements of the brain circadian clock. Trends Neurosci 28:145-151.

Arendt J (2009) Managing jet lag: some of the problems and possible new solutions. Sleep Med Rev 13:249-256.

Biello SM (2009) Circadian clock resetting in the mouse changes with age. Age 31:293-303.

Buhr ED, Yoo SH, Takahashi JS (2010) Temperature as a universal resetting cue for mammalian circadian oscillators. Science 330:379-385.

Castanon-Cervantes O, Wu M, Ehlen JC, Paul K, Gamble KL, Johnson RL, Besing RC, Menaker M, Gewirtz AT, Davidson AJ (2010) Dysregulation of inflammatory responses by chronic circadian disruption. J Immunol 185:5796-5805.

Conlon M, Lightfoot N, Kreiger N (2007) Rotating shift work and risk of prostate cancer. Epidemiology 18:182-183.

Davidson AJ, Sellix MT, Daniel J, Yamazaki S, Menaker M, Block GD (2006) Chronic jet-lag increases mortality in aged mice. Curr Biol 16:R914R916.

Davidson AJ, Yamazaki S, Arble DM, Menaker M, Block GD (2008) Resetting of central and peripheral circadian oscillators in aged rats. Neurobiol Aging 29:471-477.

Davidson AJ, Castanon-Cervantes O, Leise TL, Molyneux PC, Harrington ME (2009) Visualizing jet lag in the mouse suprachiasmatic nucleus and peripheral circadian timing system. Eur J Neurosci 29:171-180.

Dibner C, Schibler U, Albrecht U (2010) The mammalian circadian timing system: organization and coordination of central and peripheral clocks. Annu Rev Physiol 72:517-549.

Duncan MJ, Herron JM, Hill SA (2001) Aging selectively suppresses vasoactive intestinal peptide messenger RNA expression in the suprachiasmatic nucleus of the Syrian hamster. Brain Res Mol Brain Res 87:196-203.

Egli M, Bertram R, Sellix MT, Freeman ME (2004) Rhythmic secretion of prolactin in rats: action of oxytocin coordinated by vasoactive intestinal polypeptide of suprachiasmatic nucleus origin. Endocrinology 145:3386-3394.

Evans JA, Leise TL, Castanon-Cervantes O, Davidson AJ (2011) Intrinsic regulation of spatiotemporal organization within the suprachiasmatic nucleus. PLoS One 6:e15869.

Farajnia S, Michel S, Deboer T, vanderLeest HT, Houben T, Rohling JH, Ramkisoensing A, Yasenkov R, Meijer JH (2012) Evidence for neuronal desynchrony in the aged suprachiasmatic nucleus clock. J Neurosci 32: 5891-5899.

Gerhold LM, Horvath TL, Freeman ME (2001) Vasoactive intestinal peptide fibers innervate neuroendocrine dopaminergic neurons. Brain Res 919:48-56.

Gubin DG, Gubin GD, Waterhouse J, Weinert D (2006) The circadian body temperature rhythm in the elderly: effect of single daily melatonin dosing. Chronobiol Int 23:639-658.

Han S, Yu FH, Schwartz MD, Linton JD, Bosma MM, Hurley JB, Catterall WA, de la Iglesia $\mathrm{HO}$ (2012) $\mathrm{Na}_{\mathrm{V}} 1.1$ channels are critical for intercellular communication in the suprachiasmatic nucleus and for normal circadian rhythms. Proc Natl Acad Sci U S A 109:E368-E377.

Kalló I, Kalamatianos T, Piggins HD, Coen CW (2004) Ageing and the diurnal expression of mRNAs for vasoactive intestinal peptide and for the VPAC2 and PAC1 receptors in the suprachiasmatic nucleus of male rats. J Neuroendocrinol 16:758-766.
Kalsbeek A, Perreau-Lenz S, Buijs RM (2006) A network of (autonomic) clock outputs. Chronobiol Int 23:521-535.

Karatsoreos IN, Bhagat S, Bloss EB, Morrison JH, McEwen BS (2011) Disruption of circadian clocks has ramifications for metabolism, brain, and behavior. Proc Natl Acad Sci U S A 108:1657-1662.

Kolker DE, Fukuyama H, Huang DS, Takahashi JS, Horton TH, Turek FW (2003) Aging alters circadian and light-induced expression of clock genes in golden hamsters. J Biol Rhythms 18:159-169.

Kondratova AA, Kondratov RV (2012) The circadian clock and pathology of the ageing brain. Nat Rev Neurosci 13:325-335.

Li H, Satinoff E (1995) Changes in circadian rhythms of body temperature and sleep in old rats. Am J Physiol 269:R208-R214.

Lucassen PJ, Hofman MA, Swaab DF (1995) Increased light intensity prevents the age related loss of vasopressin-expressing neurons in the rat suprachiasmatic nucleus. Brain Res 693:261-266.

Lupi D, Semo M, Foster RG (2012) Impact of age and retinal degeneration on the light input to circadian brain structures. Neurobiol Aging.

Madeira MD, Sousa N, Santer RM, Paula-Barbosa MM, Gundersen H) (1995) Age and sex do not affect the volume, cell numbers, or cell size of the suprachiasmatic nucleus of the rat: an unbiased stereological study. J Comp Neurol 361:585-601.

Mahoney MM (2010) Shift work, jet lag, and female reproduction. Int J Endocrinol 2010:813764.

Mazza M, Della Marca G, De Risio S, Mennuni GF, Mazza S (2004) Sleep disorders in the elderly. Clin Ter 155:391-394.

Mohawk JA, Green CB, Takahashi JS (2012) Central and peripheral circadian clocks in mammals. Annu Rev Neurosci 35:445-462.

Monk TH (2005) Aging human circadian rhythms: conventional wisdom may not always be right. J Biol Rhythms 20:366-374.

Moore RY (1996) Neural control of the pineal gland. Behav Brain Res 73: $125-130$.

Morin LP (2007) SCN organization reconsidered. J Biol Rhythms 22:3-13.

Morris CJ, Aeschbach D, Scheer FA (2012) Circadian system, sleep and endocrinology. Mol Cell Endocrinol 349:91-104.

Mrosovsky N (1999) Masking: history, definitions, and measurement. Chronobiol Int 16:415-429.

Nagano M, Adachi A, Nakahama K, Nakamura T, Tamada M, MeyerBernstein E, Sehgal A, Shigeyoshi Y (2003) An abrupt shift in the day/ night cycle causes desynchrony in the mammalian circadian center. J Neurosci 23:6141-6151.

Nakamura TJ, Nakamura W, Yamazaki S, Kudo T, Cutler T, Colwell CS, Block GD (2011) Age-related decline in circadian output. J Neurosci 31:10201-10205.

Nakamura W, Yamazaki S, Takasu NN, Mishima K, Block GD (2005) Differential response of Period 1 expression within the suprachiasmatic nucleus. J Neurosci 25:5481-5487.

Palomba M, Nygård M, Florenzano F, Bertini G, Kristensson K, Bentivoglio M (2008) Decline of the presynaptic network, including GABAergic terminals, in the aging suprachiasmatic nucleus of the mouse. J Biol Rhythms 23:220-231.

Pulivarthy SR, Tanaka N, Welsh DK, De Haro L, Verma IM, Panda S (2007) Reciprocity between phase shifts and amplitude changes in the mammalian circadian clock. Proc Natl Acad Sci U S A 104:20356-20361.

Roberts DE, Killiany RJ, Rosene DL (2012) Neuron numbers in the hypothalamus of the normal aging rhesus monkey: stability across the adult lifespan and between the sexes. J Comp Neurol 520:1181-1197.

Rohling JH, vanderLeest HT, Michel S, Vansteensel MJ, Meijer JH (2011) Phase resetting of the mammalian circadian clock relies on a rapid shift of a small population of pacemaker neurons. PLoS One 6:e25437.

Roozendaal B, van Gool WA, Swaab DF, Hoogendijk JE, Mirmiran M (1987) Changes in vasopressin cells of the rat suprachiasmatic nucleus with aging. Brain Res 409:259-264.

Scheer FA, Hilton MF, Mantzoros CS, Shea SA (2009) Adverse metabolic and cardiovascular consequences of circadian misalignment. Proc Natl Acad Sci U S A 106:4453-4458.

Sujino M, Furukawa K, Koinuma S, Fujioka A, Nagano M, Iigo M, Shigeyoshi Y (2012) Differential entrainment of peripheral clocks in the rat by glucocorticoid and feeding. Endocrinology 153:2277-2286.

Takahashi JS, Hong HK, Ko CH, McDearmon EL (2008) The genetics of mammalian circadian order and disorder: implications for physiology and disease. Nat Rev Genet 9:764-775. 
Turek FW, Penev P, Zhang Y, van Reeth O, Zee P (1995) Effects of age on the circadian system. Neurosci Biobehav Rev 19:53-58.

Valentinuzzi VS, Scarbrough K, Takahashi JS, Turek FW (1997) Effects of aging on the circadian rhythm of wheel-running activity in C57BL/6 mice. Am J Physiol 273:R1957-R1964.

Van der Zee EA, Jansen K, Gerkema MP (1999) Severe loss of vasopressinimmunoreactive cells in the suprachiasmatic nucleus of aging voles coincides with reduced circadian organization of running wheel activity. Brain Res 816:572-579.

van Gool WA, Mirmiran M (1986) Effects of aging and housing in an enriched environment on sleep-wake patterns in rats. Sleep 9:335-347.

vanderLeest HT, Rohling JH, Michel S, Meijer JH (2009) Phase shifting capacity of the circadian pacemaker determined by the SCN neuronal network organization. PLoS One 4:e4976.

Vansteensel MJ, Yamazaki S, Albus H, Deboer T, Block GD, Meijer JH (2003) Dissociation between circadian Perl and neuronal and behavioral rhythms following a shifted environmental cycle. Curr Biol 13:1538-1542.

Vujovic N, Davidson AJ, Menaker M (2008) Sympathetic input modulates, but does not determine, phase of peripheral circadian oscillators. Am J Physiol Regul Integr Comp Physiol 295:R355-360.

Wise PM, Weiland NG, Scarbrough K, Lloyd JM (1994) Changing diurnal and pulsatile rhythms during aging. Neurobiol Aging 15:503-507.
Wyse CA, Coogan AN (2010) Impact of aging on diurnal expression patterns of CLOCK and BMAL1 in the mouse brain. Brain Res 1337:21-31.

Yamazaki S, Numano R, Abe M, Hida A, Takahashi R, Ueda M, Block GD, Sakaki Y, Menaker M, Tei H (2000) Resetting central and peripheral circadian oscillators in transgenic rats. Science 288:682-685.

Yamazaki S, Straume M, Tei H, Sakaki Y, Menaker M, Block GD (2002) Effects of aging on central and peripheral mammalian clocks. Proc Natl Acad Sci U S A 99:10801-10806.

Yan L (2011) Structural and functional changes in the suprachiasmatic nucleus following chronic circadian rhythm perturbation. Neuroscience 183:99-107.

Yan L, Silver R (2004) Resetting the brain clock: time course and localization of $\mathrm{mPER} 1$ and $\mathrm{mPER} 2$ protein expression in suprachiasmatic nuclei during phase shifts. Eur J Neurosci 19:1105-1109.

Yoo SH, Yamazaki S, Lowrey PL, Shimomura K, Ko CH, Buhr ED, Siepka SM, Hong HK, Oh WJ, Yoo OJ, Menaker M, Takahashi JS (2004) PERIOD2::LUCIFERASE real-time reporting of circadian dynamics reveals persistent circadian oscillations in mouse peripheral tissues. Proc Natl Acad Sci U S A 101:5339-5346.

Yoshikawa T, Yamazaki S, Menaker M (2005) Effects of preparation time on phase of cultured tissues reveal complexity of circadian organization. J Biol Rhythms 20:500-512. 